\title{
The Polymerization of 2,4-Hexadiene. III. Cationic Polymerizability of 2,4-Hexadiene Isomers
}

\author{
Mikiharu Kamachi, Kazuhiro Matsumura, \\ and Shunsuke Murahashi \\ Faculty of Science, Osaka University, Toyonaka, Japan.
}

(Received February 28, 1970)

\begin{abstract}
The cationic homo- and copolymerizations of geometrical isomers of 2,4-hexadiene by various catalysts in toluene or nitroethane were investigated.

The polymers obtained from three geometrical isomers were all of the trans-1,4 structure and the polymerizability of the isomeric monomers increased in the order: trans-trans $<$ cis-cis < cis-trans isomer. With an estimation of activation parameters for this polymerization, the energy diagram for the cationic polymerization of these isomers was shown and reasons for the difference in cationic polymerizabilities were suggested.

The monomer reactivity ratio of cis-trans to trans-trans isomers decreased from 1.51 in toluene (nonpolar solvent) to 1.43 in nitroethane (polar solvent). The rate of polymerization is greater in nitroethane than in toluene, as expected for cationic propagation. In the nonpolar solvent, toluene, the decrease of the monomer reactivity ratio and the increase in the polymerization rate for the $\mathrm{WCl}_{6}$ catalyst in comparison with that of $\mathrm{BF}_{3} \mathrm{O}\left(\mathrm{C}_{2} \mathrm{H}_{5}\right)_{2}$, suggest that the propagating cation obtained from $\mathrm{WCl}_{6}$ has a freer ionic character than that from $\mathrm{BF}_{3} \mathrm{O}\left(\mathrm{C}_{2} \mathrm{H}_{5}\right)_{2}$.

The molecular weight of the polymers increased in the order: cis-cis <cis-trans $<$ trans-trans, and this might be ascribed to an easy monomer transfer reaction caused in the most unstable cis-cis isomer and an easy self-termination of the propagating cation obtained from the most unstable cis-cis isomer.

KEY WORDS 2,4-Hexadiene/Cationic Polymerization / Copolymerization / Propagation Cation / Energy Diagram / Free-Ionic Character / Monomer Transfer/
\end{abstract}

We previously reported on the cationic polymerization of acetalazine $\left(\mathrm{CH}_{3}-\mathrm{CH}=\mathrm{N}-\mathrm{N}=\mathrm{CH}-\right.$ $\mathrm{CH}_{3}$ ) and the formation of an oligomer (trimer or tetramer) without the formation of a high polymer. ${ }^{1}$ It was pointed out from the kinetic study that a termination reaction caused by the hydrazine derivatives formed in its polymerization was responsible. It was also reported in a patent that only an oligomer was obtained in the polymerization of 2,4-hexadiene, a hydrocarbon analog of acetalazine.

A consideration of these facts suggested that there may be common factors which terminate the polymerization of acetalazine other than the hydrazine derivative. Thus we tried to polymerize 2,4-hexadiene in order to compare its reactivity with that of acetalazine and found that high polymers were obtainable from coordi- nation and cationic polymerization, but that only an oligomer resulted from anionic polymerization. $^{3}$

On studying the polymerizability of isomers of this monomer, we noticed that the coordination polymerizability decreased in the order trans-trans $>c i s$-trans $>c i s-c i s$ isomer and that a crystalline polymer was obtained from the trans-trans isomer, ${ }^{4}$ while both the cistrans and cis-cis isomers gave only amorphous polymers. These results were rationalized by a coordination of the cisoid form with a catalyst only in the trans-trans isomer. ${ }^{3}$

We also found that the structure of the polymer obtained from each geometrical isomer with the same cationic catalyst was almost the same and that the cationic polymerizability of each isomer decreased in the order cis- 
trans $>$ cis-cis $>$ trans-trans isomer. This suggests that 2,4-hexadiene may be a key monomer in the elucidation of the mechanism of cationic polymerization.

Thus, in this paper, the differences in cationic polymerizabilities of the 2,4-hexadiene isomers were studied in some detail.

\section{EXPERIMENTAL}

\section{Materials}

2,4-Hexadiene Isomers. Both the trans-trans and cis-trans isomers were synthesized by crotonaldahyde and ethylmagnesium bromide, followed by hydrolysis and subsequent dehydration of the alcohol. ${ }^{15}$ In this reaction, an appreciable quantity of 1,3-hexadiene was produced as a by-product but could be separated from 2,4-hexadiene by distillation. The cis-cis isomer could not be produced by this method.

The cis-cis isomer was prepared by the isomerization of 1-cis-4-hexadiene (supplied from the Toyo Rayon Co. Ltd) with $\mathrm{Fe}(\mathrm{acac})_{3}-\mathrm{Et}_{3} \mathrm{Al}$ as catalyst. ${ }^{6,7}$

The reaction was carried out in a nitrogen atmosphere at $60^{\circ} \mathrm{C}$ at a molar ratio of $\mathrm{Al} / \mathrm{Fe}=4$. The isomer ratio of the product was trans-trans: cis-trans: cis-cis=1:6:3. The cis-cis isomer was separated from the mixture of isomers by fractional distillation through a spinning band column.

Purities of the isomers were determined by gas chromatography (column: Silicon DC 550 on Celite $545,4.5 \mathrm{~m}, 80^{\circ} \mathrm{C}$; carrier gas, $\mathrm{He}$ ) and it was found that each of the isomers thus obtained had an isomeric purity of greater than 90\%. These isomers were dried over calcium hydride before use in polymerization.

\section{Solvent}

Toluene was washed successively with sulfuric acid, water, dilute aqueous sodium hydroxide and water, dried over calcium chloride, refluxed over sodium metal and distilled. Nitroethane was purified by fractional distillation and dried over calcium sulfate.

\section{Catalysts}

Borontrifluoride etherate, stannic chloride and titanium terachloride were purified by distillation before use. Antimony pentachloride was distilled under reduced pressure and tungsten hexachloride was purified by sublimation in a high vacuum. Each sample was stored in an ampoule flushed with nitrogen.

\section{Polymerization Procedure}

The polymerization was carried out under a nitrogen atmosphere. In a $30 \mathrm{ml}$ polymerization tube equipped with a mechanical stirrer and a self-sealing rubber cap, monomer and solvent were charged and the mixture was brought to the polymerization temperature. The polymerization was initiated by adding a toluene solution of a catalyst through the rubber cap in the case of $\mathrm{BF}_{3} \mathrm{OEt}_{2}, \mathrm{SnCl}_{4}$ and $\mathrm{TiCl}_{4}$. In the case of $\mathrm{SbCl}_{5}$ and $\mathrm{WCl}_{6}$, the catalysts were used without dilution. The conversion was determined gas chromatographically by measuring the residual monomer, using toluene as an internal standard.

After a given period of time the polymerization was stopped by adding methanol containing a small amount of ammonia. The polymer was purified by reprecipitating it from a benzene solution with methanol.

\section{Measurements}

Viscosities were measured in a toluene solution at $30^{\circ} \mathrm{C}$. NMR spectra were recorded on a Varian A60 spectrometer. Infrared spectra were measured in a film state on a Nippon Bunko IR-S spectrometer. A Shimazu Gas Chromatograph GC-2C was used with a $4.5-\mathrm{m}$ column of Silicon DC on Celite 545 at $80^{\circ} \mathrm{C}$, $\mathrm{He}$ gas at $24 \mathrm{ml} / \mathrm{min}$.

\section{RESULTS}

It is necessary to check the isomerization of monomers during polymerization in order to establish the method for comparing the reactivity of isomeric monomers. For the trans-trans and cis-trans isomers, which are energetically more stable, no isomerization was observed with catalysts of the Friedel-Crafts type. For the cis-cis isomer, no isomerization was observed with $\mathrm{BF}_{3} \mathrm{OEt}_{2}$ but some isomerization with $\mathrm{SnCl}_{4}$ did take place.

The structure of the polymers obtained from these isomers with a given catalyst was determined by their infrared and NMR spectra. The predominant structures were of the trans-1,4-type 
in all cases, suggesting that the propagating carbonium ions may have the same structure.

In a copolymerization of $\beta$-substituted vinyl ethers, Fueno and coworkers ${ }^{8}$ proposed a convenient method for evaluating the monomer reactivity ratios of isomeric monomers, assuming the identity of carbonium ions during propagation. If the propagating carbonium ions of isomers are identical, a $\log$ - $\log$ plot of the relative concentrations $[\mathrm{M}] /[\mathrm{M}]_{0}$ of the two monomers should be a straight line passing through the origin.

A $\log -\log$ plot of the relative monomer concentrations is shown in Figure 1, where a $1: 1$

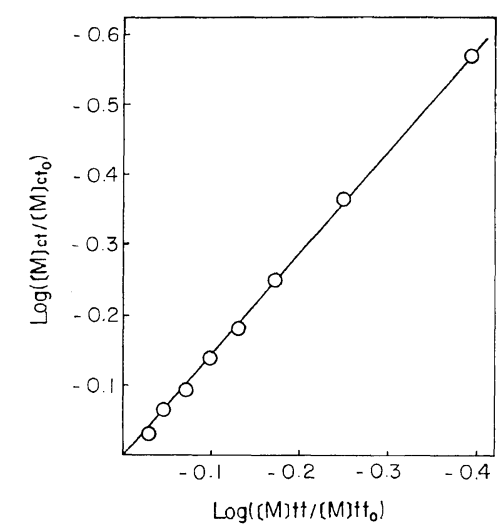

Flgure 1. The $\log -\log$ plot of the relative monomer concentration: [Monomer], $0.87 \mathrm{~mol} / l ;\left[\mathrm{SnCl}_{4}\right.$ ], $0.067 \mathrm{~mol} / l$.

mixture of trans-trans and cis-trans isomer was copolymerized at $0^{\circ} \mathrm{C}$ using $\mathrm{SnCl}_{4}$. Here, $[\mathrm{M}] c t_{0}$ and $[\mathrm{M}] c t$ are respectively the initial concentration of the cis-trans isomer, and its concentration at a given polymerization time. Figure 1 clearly shows that the straight line passes through the origin. The same result was obtained in the copolymerization between the cis-cis and cis-trans isomers. The plot of $\ln [\mathrm{M}]_{0} /[\mathrm{M}]$ against time in each geometrical isomer was linear in the copolymerization system. Therefore, the propagating carbonium ions for these copolymerizations are identical, as anticipated from the structure of the polymer obtained. The slope of these straight lines should correspond to the monomer reactivity ratios of the isomers, judging from the fact that the molecular weights of the polymers obtained are sufficiently high for the effect of initiation, termination and transfer reactions to be ignored.

The monomer reactivity ratios evaluated by this method are shown in Table I. In the

Table I. Monomer reactivity ratios

(a)

\begin{tabular}{lcc}
\hline \multicolumn{1}{c}{ Catalyst } & $\mathrm{ct} / \mathrm{tt}$ & $\mathrm{ct} / \mathrm{cc}$ \\
\hline $\mathrm{BF}_{3} \mathrm{OEt}_{2}$ & $1.51 \pm 0.02$ & $1.31 \pm 0.01$ \\
$\mathrm{SnCl}_{4}$ & $1.47 \pm 0.02$ & - \\
$\mathrm{TiCl}_{4}$ & $1.49 \pm 0.04$ & - \\
$\mathrm{SbCl}_{5}$ & $1.47 \pm 0.03$ & - \\
$\mathrm{WCl}_{6}$ & $1.30 \pm 0.03$ & - \\
\hline
\end{tabular}

Solvent, toluene at $0^{\circ} \mathrm{C}$

(b)

\begin{tabular}{ccc}
\hline Catalyst & $\mathrm{ct} / \mathrm{tt}$ & $\mathrm{ct} / \mathrm{cc}$ \\
\hline $\mathrm{BF}_{3} \mathrm{OEt}_{2}$ & $1.43 \pm 0.02$ & $1.20 \pm 0.02$ \\
\hline
\end{tabular}

Solvent, nitroethane at $0^{\circ} \mathrm{C}$

copolymerization of the cis-cis with cis-trans isomers, the monomer reactivity ratios were evaluated only in the case of $\mathrm{BF}_{3} \mathrm{OEt}_{2}$, because some isomerization of the cis-cis isomer was shown to occur competitively in polymerization catalyzed by other metal halides. The monomer reactivity ratio is 1.51 for the cis-trans and trans-trans isomer pair and 1.31 for the cistrans and cis-cis isomer system. These values show that the cationic polymerizability decreases in the order cis-trans $>$ cis-cis $>$ trans-trans.

The energy differences of the isomers in the initial state at $0^{\circ} \mathrm{C}$ have been shown to be as follows. ${ }^{9}$ These data suggest that the thermodynamic stability of the isomers increases in the

$\partial G($ cis-trans $)-\partial G($ trans - trans $)=0.43 \mathrm{kcal} / \mathrm{mol}$ $\partial G($ cis-cis $)-\partial G($ cis-trans $)=1.20 \mathrm{kcal} / \mathrm{mol}$

order $c i s-c i s>c i s-t r a n s>$ trans-trans. The differences of the activation enthalpy and the activation entropy were determined from the variation of the monomer reactivity ratios in the temperature range $0^{\circ} \mathrm{C}$ to $-78^{\circ} \mathrm{C}$, as shown in Figure 2 and Table II. The differences in the activation free energy at $0^{\circ} \mathrm{C}$ are estimated to be as follows. 
M. Kawachi, K. Matsumura, and S. Murahashi

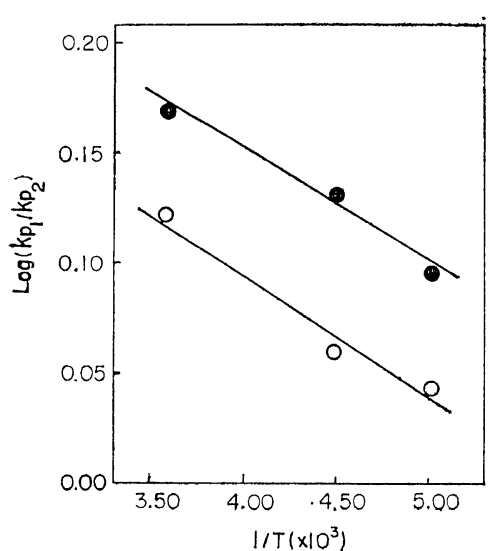

Figure 2. Arrhenius plot of the monomer reactivity ratios: - cis-trans/trans-trans; O, cis-trans/ cis-cis.

Table II. Activation parameters

$\delta \Delta H^{\neq}, \mathrm{kcal} / \mathrm{mol} \delta \Delta S^{\neq}$, e.u. $\delta \Delta G^{\neq}, \mathrm{kcal} / \mathrm{mol}$

\begin{tabular}{llll}
\hline $\mathrm{ct} / \mathrm{tt}$ & $0.22 \pm 0.03$ & $1.58 \pm 0.12$ & -0.21 \\
$\mathrm{ct} / \mathrm{cc}$ & $0.26 \pm 0.05$ & $1.46 \pm 0.20$ & -0.14 \\
\hline
\end{tabular}

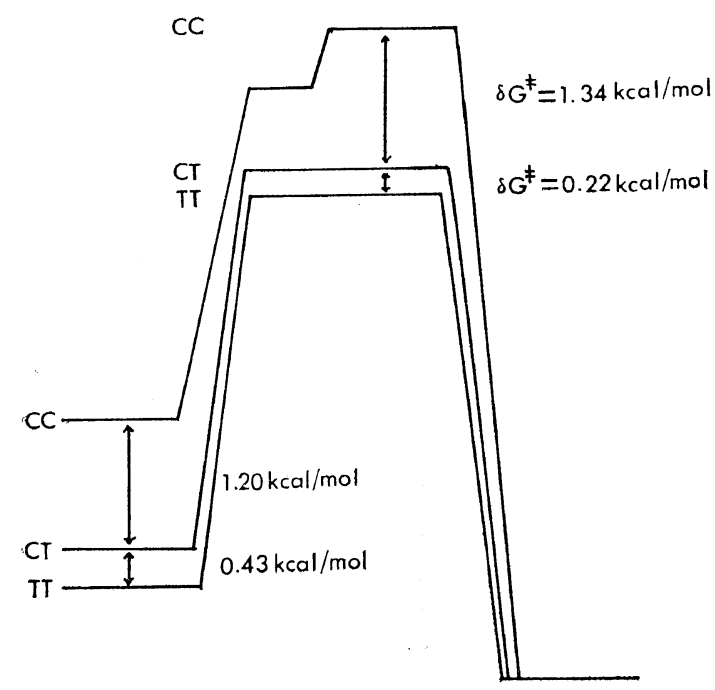

Figure 3. Energy diagram for the polymerization of 2,4-hexandiene at $0^{\circ} \mathrm{C}$.

$$
\begin{aligned}
\Delta G^{\neq}(\text {cis }- \text { trans })-\Delta G^{\neq}(\text {trans-trans }) & = \\
& -0.21 \mathrm{kal} / \mathrm{mol} \\
\Delta G^{\neq}(\text {cis }-c i s)-\Delta G^{\neq}(\text {cis-trans })= & 0.14 \mathrm{kal} / \mathrm{mol}
\end{aligned}
$$

The energy diagram of this polymerization is shown in Figure 3. Both the activation enthalpy and the activation entropy of the cis-trans isomer are greater than those of the other isomer, as shown in Table II. It may also be seen that the entropy effect makes a larger contribution than the enthalpy effect at $0^{\circ} \mathrm{C}$.

\section{DISCUSSION}

The energy difference $(0.43 \mathrm{kcal})$ between the cis-trans and trans-trans isomers in the ground state is larger than that $(0.22 \mathrm{kcal} / \mathrm{mol})$ in the transition state. Thus, the trans-trans isomer requires a larger free energy of activation than the cis-trans isomer; that is, the polymerizability of the cis-trans isomer is greater than that of the trans-trans isomer.

We assume the formation of the propagating carbonium ions as follows.

In the case of the trans-trans isomer, the propagating carbonium ion formed by the attack<smiles>C/C=C\C=C/C=C\C</smiles><smiles>CC=CC=CC</smiles><smiles>CC=CC</smiles><smiles>CC=CCC</smiles><smiles>C/C=C\C=C/C=C\C</smiles>

Scheme 1 
of the cation on the monomer is a transoid-type. In the cis-trans isomer, the type of carbonium ion formed can be either transoid- or cisoid-type, depending on the attacking position of the double bond. However, the formation of the transoidtype carbonium ion is considered to be more favorable for the following two reasons. Firstly, the transiod-type carbonium ion may be energetically more stable than the cisoid-type carbonium ion, judging from the difference between the heats of hydrogenation of trans- and cis-2butenes. ${ }^{10}$ Secondly, a complex formation with $\mathrm{Ag}^{+}$in a cis position is more favorable than in a trans position, as deduced from the value of equilibrium constant of a complex formation of butene-2 with $\mathrm{Ag}^{+} .{ }^{11}$ Thus, the propagating cations of the trans-trans and cis-trans isomers are identical.

For the cis-cis isomer, the primary formation of a cisoid-type carbonium ion occurs, but in order for the identical carbonium ion to be formed it is necessary that the cisoid-tpye carbonium ion rotates to give the more stable transiod-type ion. The activation enthalpy of this rotation in substituted allylic cations has been reported to be about $4-5 \mathrm{kal} / \mathrm{mol}^{12}$ Thus rotation of the carbonium ion before propagation is unlikely. This assumption reasonably explains why the more unstable cis-cis isomer in the initial state is less reactive than the more stable cis-trans isomer.

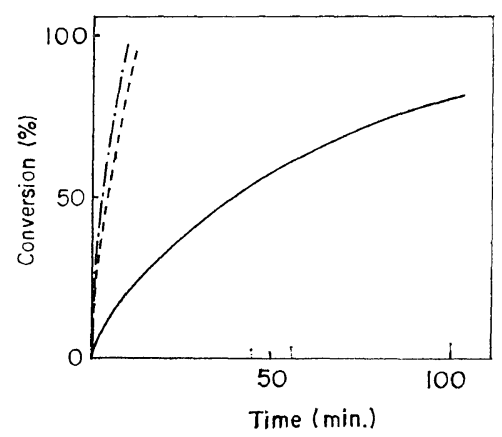

Figure 4. Time conversion curve of the cis-trans isomer: -, $\mathrm{BF}_{3} \mathrm{OEt}_{2}$ in toluene; $\left[\mathrm{BF}_{3} \mathrm{OEt}_{2}\right]$, $0.067 \mathrm{~mol} / l$; [Monomer], $0.87 \mathrm{~mol} / l ; \cdots,-\mathrm{BF}_{3} \mathrm{OEt}_{2}$ in nitroethane; $\left[\mathrm{BF}_{3} \mathrm{OEt}_{2}\right.$ ], $0.067 \mathrm{~mol} / l$; [Monomer], $0.87 \mathrm{~mol} / l ;-\cdot-\cdot, \mathrm{WCl}_{6}$ in totuene; [ $\mathrm{WCl}_{6}$ ], 0.067 $\mathrm{mol} / l$, [Monomer], $0.87 \mathrm{~mol} / l$.
The variations in monomer reactivity ratios. with different catalysts or solvents are shown in Table I. A comparison of the monomer reactivity ratios in toluene with those in nitroethane in the case of $\mathrm{BF}_{3} \mathrm{OEt}_{2}$ reveals that the monomer reactivity ratios decrease from 1.51 in toluene to 1.43 in nitroethane for the cis-trans/ trans-trans system, and from 1.31 to 1.20 for the cis-trans/cis-cis system. The time conversion curve of the pure cis-trans isomer is shown in Figure 4. The rate of the polymerization increases appreciably in nitroethane and this may correspond to the increase in the freeionic character of propagating cations in polar solvents. In the copolymerization, therefore, the rate of the polymerization of each isomer increases in a high dielectric constant medium: the selectivity of the propagating cation to monomers and hence the monomer reactivity ratios decrease accordingly.

The dependence of monomer reactivity ratios on different catalysts in toluene was also investigated (Table I). The monomer reactivity ratio between the cis-trans and trans-trans isomers decreases to 1.30 in the case of $\mathrm{WCl}_{6}$. The rate of homopolymerization of the cis-trans isomer by $\mathrm{WCl}_{6}$ also increases (Figure 4). These results might be ascribed to an increase in the free-ionic character of the propagating cation because of a larger gegenanion derived from $\mathrm{WCl}_{6}$. This corresponds to the polymerization of styrene by $\mathrm{ReCl}_{5}$, reported previously by one of the authors. ${ }^{13}$ In this case, the rate constant of propagation was appreciably larger than that with $\mathrm{SnCl}_{4}$, a catalyst containing a smaller gegenion.

The intrinsic viscosities of the polymers obtained from the three isomers and their mixtures are shown in Table III. The degree of polymerization is lowest in the polymer obtained from the cis-cis isomer. In the case of mixtures of the

Table III. Intrinsic viscosities of the polymers

\begin{tabular}{ll} 
Isomers & {$[\eta]$} \\
\hline $\mathrm{tt}: \mathrm{ct}=2: 1$ & 0.26 \\
$\mathrm{tt}: \mathrm{ct}=0.8: 1$ & 0.21 \\
$\mathrm{ct}$ & 0.16 \\
$\mathrm{cc}$ & 0.16
\end{tabular}

Catalyst, $\mathrm{BF}_{3} \mathrm{OEt}_{2} ;$ toluene at $30^{\circ} \mathrm{C}$ 
trans-trans and cis-trans isomers, the higher the trans-trans isomer content, the higher is the molecular weight of the polymer. These results may suggest that the monomer transfer is easiest in the most unstable cis-cis isomer, and the self-termination is easiest in the propagating cation obtained from the most unstable cis-cis isomer.

Acknowledgment. The authors are grateful to the Toyo Rayon Co., Ltd. for supplying 1-cis-4hexadiene. They would also like to express their appreciation to Professor J. K. Stille and Dr. S. Nozakura for useful discussions and criticism.

\section{REFERENCES}

1. N. Wakabayashi, M. Kamachi, and S. Murahashi, paper presented at the 13th Meeting of High Polymers, Kobe, 1967.

2. W. H. Carothers, Ind. Eng. Chem., 26, 30 (1934).

3. S. Murahashi, M. Kamachi, and N. Wakaba- yashi, J. Polym. Sci., Part B, 7, 135 (1969).

4. M. Kamachi, N. Wakabayashi, S. Murahashi, to be published.

5. R. Adams and T. A. Geissman, J. Amer. Chem. Soc., 61, 2085 (1939).

6. G. Hata and D. Aoki, J. Org. Chem., 32, 3754 (1967).

7. M. Iwamoto and S. Yuguchi, Bull. Chem. Soc. Japan, 39, 2001 (1965).

8. N. Okuyama, N. Asami, and T. Fueno, Preprint, 17th Symposium on Polymer Chemistry, Matsuyama, 1968, p 215.

9. V. E. Doering and H. G. Hauthar, J. Prakt. Chem., 24, 27 (1964).

10. C. B. Kistiakowsky, J. R. Ruhoff, H. A. Smith, and W. E. Vaughan, J. Amer. Chem. Soc., 57, 876 (1935).

11. M. A. Muhs and F. T. Weiss, ibid., 84, 4697 (1962).

12. G. Olah and J. M. Bollinger, ibid., 90, 6082 (1968).

13. M. Kamachi and H. Miyama, J. Polym. Sci., Part A, 3, 1337 (1965). 